\title{
Incorporação de Nanomateriais e emulsão de ceras no desenvolvimento de papéis multicamadas
}

\author{
Incorporation of nanomaterials and emulsion of waxes in the \\ development of multilayer papers
}

\section{Lays Camila Matos ${ }^{1 *}$, Vinícius Dutra Rompa ${ }^{1}$, Renato Ausgusto Pereira Damásio² José Manoel Marconcini ${ }^{3}$, Gustavo Henrique Denzin Tonoli ${ }^{1}$}

\begin{abstract}
RESUMO
Neste trabalho, objetivou-se avaliar os efeitos do revestimento por deposição de camadas de nanofibrilas celulósicas nanoargila e emulsões de cera de abelha e carnaúba na gramatura, espessura e permeabilidade ao vapor de água de papéis kraft. As suspensões foram depositadas por espatulagem sobre o papel kraft de gramatura $60 \mathrm{~g} / \mathrm{m}^{2}$ até atingir gramatura de $80 \mathrm{~g} / \mathrm{m}^{2}$, onde foram produzidos tratamentos contendo apenas camadas de nanofibrilas celulósicas (NF) e camadas de nanoargila (MMT), tratamentos com camadas de nanofibrilas celulósicas sobrepostas por camadas de emulsão de cera de carnaúba (NF/EC) e de abelha (NF/EA), tratamentos com camadas de nanoargila também sobrepostas por camadas de emulsão de ceras de carnaúba (MMT/EC) e de abelha (MMT/EA) e tratamentos apenas com as emulsões das ceras (EC e EA). Os tratamentos foram comparados com os papéis kraft de $60 \mathrm{~g} / \mathrm{m}^{2}$ (Ref 60 ) e de $80 \mathrm{~g} / \mathrm{m}^{2}$ (Ref 80). As imagens eletrônicas de varredura da superfície e da seção transversal dos papéis dos tratamentos mostraram uma redução significativa dos espaços vazios presentes no papel referência $\left(60 \mathrm{~g} / \mathrm{m}^{2}\right)$. Com a aplicação da camada de emulsão das ceras, observou-se em EC e EA uma redução de $87 \%$ e 69\% na permeabilidade ao vapor d'água (PVA) do papel de $60 \mathrm{~g} / \mathrm{m}^{2}$, respectivamente. Quando comparados ao papel de $80 \mathrm{~g} / \mathrm{m}^{2}$ essa redução no PVA foi ainda maior ( $96 \%$ e $90 \%$, respectivamente). Quando as camadas de emulsões foram associadas a camadas de nanoargila (MMT/EC $=2,3 \mathrm{~g} \cdot \mathrm{mm} / \mathrm{dia} \cdot \mathrm{m}^{2} \cdot \mathrm{KPa}$ e MMT/EA $=4,4 \mathrm{~g} \cdot \mathrm{mm} / \mathrm{dia} \cdot \mathrm{m}^{2} \cdot \mathrm{KPa}$ ) estas obtiveram melhores resultados de PVA do que quando associadas a camadas de nanofibrilas celulósicas $\left(\mathrm{NF} / \mathrm{EC}=5,2 \mathrm{~g} \cdot \mathrm{mm} / \mathrm{dia} \cdot \mathrm{m}^{2} . \mathrm{KPa}\right.$ e NF/EA $\left.=6,8 \mathrm{~g} \cdot \mathrm{mm} / \mathrm{dia} \cdot \mathrm{m}^{2} . \mathrm{KPa}\right)$ para PVA. Os resultados mostraram como as camadas de emulsão de ceras de carnaúba e abelha atuaram na melhoria das propriedades de barreira ao vapor de água de papéis multicamadas, e como a combinação com camadas de nanofibrilas celulósicas e de nanoargila influenciam nessas propriedades.
\end{abstract}

Palavras-chave: Papéis multicamadas. Nanoestruturas. Emulsão de ceras. Propriedades de barreira ao vapor de água.

\begin{abstract}
The objective of this work was to evaluate the effects of coating by deposition of cellulose nanofibrils, nanoargila and wax emulsion of bee and carnauba in the grammage, thickness and water vapor permeability of kraft papers. The suspensions were deposited by spatulation on kraft paper of $60 \mathrm{~g} / \mathrm{m}^{2}$ until they reached a grammage of $80 \mathrm{~g} / \mathrm{m}^{2}$, where treatments containing only layers of cellulose nanofibrils (NF) and layers of nanoargila (MMT) were prepared, treatments with layers of cellulose nanofibrils overlaid by carnauba wax emulsion (NF/EC) and bee (NF/EA), treatments with layers of nanoargila also overlaid by emulsion layers of carnauba wax (MMT/EC) and bee wax (MMT/EA) and treatments with only the emulsions of the waxes (EC and EA). The treatments were compared with the kraft papers of $60 \mathrm{~g} / \mathrm{m}^{2}$ (Ref 60 ) and $80 \mathrm{~g} / \mathrm{m}^{2}$ (Ref 80 ). The scanning electron microscopy images of the surface and the cross sections showed a significant reduction of the voids present in the reference paper $\left(60 \mathrm{~g} / \mathrm{m}^{2}\right)$. With the increase of the wax emulsion layer, EC and EA showed a reduction of $87 \%$ and $69 \%$ in the water vapor permeability (PVA) of $60 \mathrm{~g} / \mathrm{m}^{2}$ paper, respectively. When compared to the $80 \mathrm{~g} / \mathrm{m}^{2}$ paper, this reduction in PVA was even higher $(96 \%$ and $90 \%$, respectively). When the layers of emulsions were associated with layers of nanoargila (MMT/EC $=2.3 \mathrm{~g} \cdot \mathrm{mm} / \mathrm{dia} \cdot \mathrm{m}^{2} . \mathrm{KPa}$ and $\left.\mathrm{MMT} / \mathrm{EA}=4.4 \mathrm{~g} \cdot \mathrm{mm} / \mathrm{dia} \cdot \mathrm{m}^{2} . \mathrm{KPa}\right)$ these obtained
\end{abstract}

1. Departamento de Ciências Florestais, Universidade Federal de Lavras - UFLA. Lavras / MG, Brasil. * Autor correspondente: laysmatos9@hotmail.com

2. Klabin SA. Telêmaco Borba / PR, Brasil.

3. Embrapa Instrumentação Agropecuária, Empresa Brasileira de Pesquisa Agropecuária. São Carlos / SP, Brasil.

Sci. For., Piracicaba, v. 47, n. 122, p. 177-191, jun. 2019 DOI: doi.org/10.18671/scifor.v47n122.01 
Matos et al. - Incorporação de Nanomateriais e emulsão de ceras no

desenvolvimento de papéis multicamadas

better PVA results than when associated with layers of celulose nanofibrils (NF/EC $=5.2 \mathrm{~g} \cdot \mathrm{mm} / \mathrm{dia} \cdot \mathrm{m}^{2} . \mathrm{KPa}$ and NF/EA $=6.8 \mathrm{~g} \cdot \mathrm{mm} / \mathrm{dia} \cdot \mathrm{m}^{2} . \mathrm{KPa}$ ) for PVA. The results showed how the emulsion layers of carnauba and bee waxes have improved the water vapor barrier properties of multilayer papers, and how the combination with layers of cellulose nanofibrils and nanoargila influences these properties.

Keywords: Multilayer papers. Nanostructures. Hydrophobicity. Wax emulsion. Water vapor barrier properties.

\section{INTRODUÇÃO}

Dentre as alternativas estudadas para minimizar o impacto ambiental dos polímeros convencionais que compõe as embalagens (polipropileno, poliestireno, policloreto de vinila (PVC), poli álcool vinílico (PVA) e polietileno tereftalato (PET), destaca-se o uso dos biopolímeros, que são biodegradáveis, renováveis e de baixo custo segundo Oijo e Ray, 2013 e Ugalde, 2014).

O papel é biodegradável, sendo por isso amplamente utilizado em aplicações de embalagens que não agridem o meio ambiente. Porém, a sua natureza hidrofílica, devido aos sítios $\mathrm{OH}$ distribuídos nos carbonos 2, 3 e 6 da unidade base de celulose (C6H10O5) e a rede porosa formada pelas fibras, limitam as propriedades de barreira ao vapor d'água do papel, e consequentemente, as suas propriedades mecânicas, quando no estado macro/microscópico e sem nenhum tipo de tratamento ou revestimento. Para minimizar este efeito, o papel é normalmente revestido com outros materiais como plástico ou alumínio, originando embalagens multicamadas. O uso de papel revestido com materiais de base biológica é uma alternativa ainda mais ecológica (HERRERA et al., 2014). Embalagens de papel revestidas com materiais de origem vegetal também são uma alternativa renovável em substituição às embalagens convencionais, representando uma solução para as embalagens do futuro, que são aquelas capazes de exercer suas funções e sem prejudicar o meio ambiente com seu descarte.

Para otimizar as propriedades de barreira do papel revestido já foram reportados na literatura diferentes materiais: polímeros e biopolímeros (ZHANG et al., 2015), ceras (DESPOND et al., 2005), argilas (KK ; KO, 2015; MIRMEHDI et al., 2018) e nanomateriais (LI et al., 2007). Quanto a melhoria de propriedades mecânicas, destacam-se os materiais nanométricos, como por exemplo as nanoargilas e as nanoestruturas celulósicas, que podem contribuir adicionalmente para a melhoria das propriedades de barreira (BENEVENTI et al., 2014; GUIMARÃES JÚNIOR et al., 2018, 2015; HULT et al., 2010; PRADO et al., 2018 SCATOLINO et al., 2017;).

Nanofibrilas celulósicas são de fácil obtenção e apresentam excelentes propriedades mecânicas (apresentam módulo elástico próximo ao do Kevlar), aliadas a uma baixa densidade $(1,6 \mathrm{~g} / \mathrm{cm} 3)$, biodegradabilidade, biocompatibilidade e possibilidade de funcionalização química devido a presença de hidroxilas superficiais (SAMIR et al., 2005). Consequentemente, as nanofibrilas de celulose têm despertado o interesse de indústrias em diferentes setores (embalagens, agricultura, alimentícia, automobilística, aeronáutica, etc.). Na indústria de embalagens o uso deste material destaca-se em aplicações de embalagens flexíveis ou rígidas (SILVA et al., 2009). Nanofibrilas celulósicas foram usadas como reforço em pasta para a produção de papel, agregando melhoria de suas propriedades mecânicas (TARRÉS et al., 2016). Mousavi et al. (2017) revestiram papel cartão com nanofibrilas celulósicas melhorando consideravelmente as propriedades de barreira deste material. Ainda, papéis revestidos com nanofibrilas celulósicas e nanopartículas de prata apresentaram ação antimicrobiana, além da redução da taxa de transmissão do vapor de água e de barreira ao óleo e aumento da resistência à tração, onde as nanofibrilas celulósicas foram essenciais para os resultados satisfatórios (AMINI et al., 2016).

As nanoargilas são partículas minerais com pelo menos uma de suas dimensões menor que $100 \mathrm{~nm}$, divididas em quatro grupos distintos. O grupo das montimorilonitas, que são argilas hidrofílicas formadas por camadas tetraédricas de sílica, preenchidas por octaedros de alumina, é o mais abundante e mais estudado (FLOODY et al., 2009). Quando associada a materiais poliméricos, a montimorilonita confere melhoria nas propriedades mecânicas (resistência à tração, flexão, resistência ao impacto, alongamento na ruptura, retardante de queima, condutividade elétrica) e também nas propriedades de barreira ao vapor de água e a gases (FLOODY et al., 2009). Wang et al. (2003) avaliaram um nanocompósito de epóxi e montimorilonita, que apresentou resistência à fratura $80 \%$ maior que o epóxi virgem. Herrera-Alonso et al. (2010) investigaram as propriedades de barreira 
de nanocompósitos de polibutilmetacrilato/nanoargila e verificaram que a permeabilidade ao gás diminuiu com o aumento da concentração de nanoargila na matriz. Bandera et al. (2016) observaram uma melhoria de $85 \%$ na taxa de transmissão de vapor de água, associada ao uso da montimorilonita, em papéis revestidos com um material a base polilactida e montimorilonita. Wang and Jing (2017) revestiram papel de embalagem convencional com nanocompósito de montimorilonita/quitosana e, também, observaram melhora das propriedades de barreira ao vapor de água.

As ceras em geral atuam como polímeros de cadeias curtas e são usadas como agentes modificadores de superfície. Possuem baixa temperatura de fusão e viscosidade, sendo a cera de polietileno, a cera de carnaúba, a parafina microcristalina e a cera de abelha as mais utilizadas, (OURIQUE et al., 2015). A cera de carnaúba, formada nas folhas e frutos das palmeiras de carnaúba (Copernicia Prunifera), apresenta temperatura de fusão entre 80 e $87^{\circ} \mathrm{C}$ (ALVES; COÊLHO, 2008) e sua composição química consiste em aproximadamente $82-85 \%$ de ésteres de ácidos graxos alifáticos e aromáticos (principalmente o ceril palmitato),10-12\% álcoois livres, 5-7\% ácidos graxos livres, 0,3-1,0\% hidrocarbonetos e 0,4-0,5\% de outros componentes (D'ALVA, 2007). Jo et al. (2014) aplicaram em maçãs a cera de carnaúba-shellac contendo óleo essencial de lemongras, e observaram uma menor perda de massa dos frutos. Despond et al. (2005) avaliaram a incorporação de uma camada de cera de carnaúba sobre o papel já revestido com quitosana e constataram a diminuição significativa da absorção de água na multicamada, devido ao caráter hidrofóbico da camada de cera. Segundo os autores os coeficientes de permeabilidade de $\mathrm{CO} 2$ e $\mathrm{O} 2$ nos papéis com cera também foram inferiores aos obtidos no papel apenas com quitosana.

A cera de abelha é proveniente da secreção de glândulas cerígenas situadas no abdome das abelhas operárias e são obtidas pelo homem através dos favos construídos por elas. É constituída por 14\% hidrocarbonetos, $67 \%$ de ésteres, $12 \%$ de ácidos, $1 \%$ são de álcoois variados e $6 \%$ de compostos não definidos (OLIVEIRA, 2017). A cera de abelha possui diversas aplicações, entre elas na indústria alimentícia, como revestimentos comestíveis e em papel para embalagem (ZHANG et al., 2014). De modo a superar as deficiências do revestimento de camada única, Zhang et al. (2014) preparam papéis com bicamadas usando várias combinações de proteínas ou polissacáridos com cera de abelha, onde o papel bicamada quitosana/cera de abelha apresentou melhor propriedade de barreira ao vapor de água. Sothornvit (2009) determinou o efeito de hidroxipropil metilcelulose (HPMC), glicerol e revestimentos de cera de abelha sobre as propriedades físicas e de permeabilidade ao vapor de água (PVA) de papéis revestidos e observaram que revestimentos à base de HPMC tiveram maior redução em PVA quando a cera de abelha foi incorporada no papel na forma de compósito HPMC-lipídico, enquanto o HPMC sozinho atuou na melhoria das propriedades mecânicas.

Desta forma, este trabalho tem como objetivo a avaliação do efeito de cada camada do papel nas propriedades de barreira ao vapor de água e na estrutura de papéis multicamadas revestidos por camadas de nanofibrilas celulósicas, montimorilonita e emulsões de ceras de carnaúba e de abelha aplicadas por espatulagem.

\section{MATERIAIS E MÉTODOS}

\section{Materiais}

Polpa comercial kraft branqueada de Eucalyptus sp. e papel kraft comercial de gramaturas de $60 \mathrm{~g} / \mathrm{m}^{2}$ e $80 \mathrm{~g} / \mathrm{m}^{2}$ fornecido pela empresa Klabin S/A; nanoargila bentonita hidrofílica (H2Al2O6Si) da família das montimorilonitas; cera de carnaúba T-1 (tipo 1-em escamas com coloração amarelo claro); cera de abelha FA 800 em escamas e de coloração branca e os emulsificantes Polissorbato 80 (Tween $80, \mathrm{EHL}=15)$ e a goma arábica $(\mathrm{EHL}=8)$.

\section{Métodos \\ Obtenção e preparo das suspensões de nanofibrilas celulósicas e nanoargila e síntese das emulsões de ceras}

Para obtenção das nanofibrilas celulósicas, das suspensões de revestimento de nanofibrilas celulósicas e nanoargila e síntese das emulsões seguiu-se os passos descritos na Figura 1, sendo que para obtenção das nanofibrilas celulósicas foram usadas metodologias de trabalhos anteriores (ARANTES et al., 2017; BUFALINO et al., 2015 FONSECA et al., 2016; GUIMARÃES JÚNIOR et al., 2015; TONOLI et al., 2016). Para a síntese das emulsões utilizou-se a metodologia adaptada de Gonçalves (2000). 


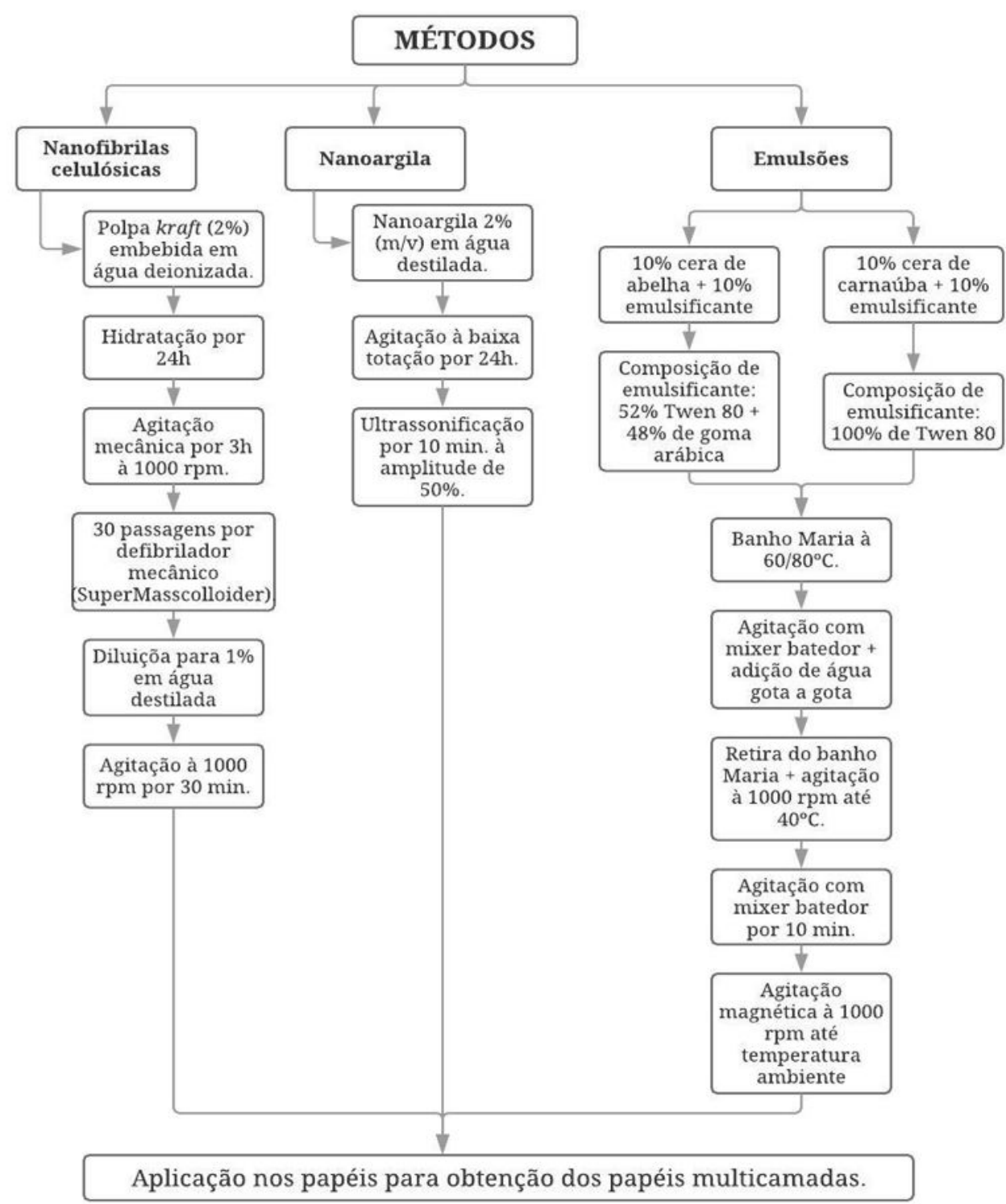

Figura 1: Esquema da metodologia seguida para o preparo das suspensões de nanofibrilas celulósicas e nanoargila e para síntese das emulsões de revestimento a serem aplicadas nos papéis.

Figure 1: Scheme of the methodology followed for preparing the suspensions of nanofibrils cellulosic and nanoargila and for synthesis of the coating emulsions to be applied in the papers.

\section{Produção dos papéis multicamadas}

Os papéis multicamadas foram produzidos com as camadas em diferentes composições, de acordo com a Tabela 1.

As camadas foram depositadas sobre o papel kraft de $60 \mathrm{~g} / \mathrm{m}^{2} \mathrm{com}$ o uso de um pincel mousse. A gramatura desejada para a embalagem multicamada foi de $80 \mathrm{~g} / \mathrm{m}^{2}$, então, foi predeterminado o peso da camada produzida por $5 \mathrm{~mL}$ de cada suspensão para estimativa do número de camadas que cada tratamento receberia, a fim de adicionar sobre o papel $20 \mathrm{~g} / \mathrm{m}^{2}$. Após a aplicação da primeira camada da solução de nanofibrilas celulósicas, o papel foi levado para estufa à temperatura de $60^{\circ} \mathrm{C}$ por 30 min e após secagem dessa camada, a próxima camada foi depositada, seguindo-se esse passo também para a deposição das camadas de montimorilonita sobre o papel, sendo que após a aplicação das emulsões de ceras o papel foi seco a temperatura ambiente, aproximadamente $26^{\circ} \mathrm{C}$.

\section{Teste de estabilidade das emulsões}

Para verificar a estabilidade das emulsões de ceras sintetizadas foi usado a centrifugação, que é a técnica mais comumente aplicada para acelerar a separação de fases e desestabilizar uma emulsão. Aproximadamente $5 \mathrm{~g}$ de cada emulsão foram pesados e submetidos, na centrífuga (Fanem, modelo: 206) 
Tabela 1: Tratamentos, número de camadas e composições dos papéis multicamadas produzidos

Table 1: Treatments, number of layers and compositions of the multilayer papers produced

\begin{tabular}{|c|c|c|c|}
\hline TRATAMENTOS & $N^{\circ}$ DE CAMADAS & COMPOSIÇÃO & $\begin{array}{c}\text { QUANTIDADE DE } \\
\text { MATERIAL ADICIONADA }\end{array}$ \\
\hline EA & 2 & PA/ECA & $24,0 \mathrm{~g} / \mathrm{m}^{2}-\mathrm{ECA}$ \\
\hline EC & 2 & PA/ECC & $24,0 \mathrm{~g} / \mathrm{m}^{2}-\mathrm{ECC}$ \\
\hline MMT & 2 & PA/SMMT & $17,0 \mathrm{~g} / \mathrm{m}^{2}-\mathrm{SMMT}$ \\
\hline $\mathrm{MMT} / \mathrm{EA}$ & 3 & PA/SMMT/ECA & $\begin{array}{l}2,4 \mathrm{~g} / \mathrm{m}^{2}-\mathrm{SMMT} \\
25,6 \mathrm{~g} / \mathrm{m}^{2}-\mathrm{ECA}\end{array}$ \\
\hline MMT/EC & 3 & PA/SMMT/ECC & $\begin{array}{l}2,4 \mathrm{~g} / \mathrm{m}^{2} \text { - SMMT } \\
19,6 \mathrm{~g} / \mathrm{m}^{2} \text { - ECC }\end{array}$ \\
\hline NF & 2 & PA/SNF & $20,3 \mathrm{~g} / \mathrm{m}^{2}-\mathrm{SNF}$ \\
\hline NF/EA & 3 & $\mathrm{PA} / \mathrm{SNF} / \mathrm{ECA}$ & $\begin{array}{r}1,2 \mathrm{~g} / \mathrm{m}^{2}-\mathrm{SNF} \\
21,8 \mathrm{~g} / \mathrm{m}^{2}-\mathrm{ECA}\end{array}$ \\
\hline NF/EC & 3 & PA/SNF/ECC & $\begin{array}{r}1,2 \mathrm{~g} / \mathrm{m}^{2}-\mathrm{SNF} \\
21,8 \mathrm{~g} / \mathrm{m}^{2}-\mathrm{ECC}\end{array}$ \\
\hline
\end{tabular}

EA: papel kraft $0 \mathrm{~g} / \mathrm{m}^{2}$ com emulsão de cera de abelha; EC: papel $\mathrm{kraft} 60 \mathrm{~g} / \mathrm{m}^{2}$ com emulsão de cera de carnaúba; MMT: papel $\mathrm{kraft} 60 \mathrm{~g} / \mathrm{m}^{2}$ com montimorilnita; MMT/EA: papel $\mathrm{kraft} 60 \mathrm{~g} / \mathrm{m}^{2} \mathrm{com}$ montimorilonita e emulsão de cera de abelha; MMT/EC: papel kraft $60 \mathrm{~g} / \mathrm{m}^{2} \mathrm{com}$ montimorilonita e emulsão de cera de carnaúba; NF: papel $\mathrm{kraft} 60 \mathrm{~g} / \mathrm{m}^{2} \mathrm{com}$ nanofibrilas celulósicas; NF/EA: papel $\mathrm{kraft} 60 \mathrm{~g} / \mathrm{m}^{2} \mathrm{com}$ nanofibrilas celulósicas e emulsão de cera de abelha; NF/EC: papel kraft $60 \mathrm{~g} / \mathrm{m}^{2} \mathrm{com}$ nanofibrilas celulósicas e emulsão de cera de carnaúba.PA: folha de papel kraft; ECA: emulsão de cera de abelha; ECC: emulsão de cera de carnaúba; SMMT: suspensão de montimorilonita; SNF: suspensão de nanofibrilas celulósicas

aos ciclos de 1600, 2400 e 3200 rpm (70, 440 e 863 g) durante 15 min em cada rotação, e em temperatura ambiente, seguindo metodologia adaptada de Almeida (2014).

\section{Avaliação da espessura e gramatura dos papéis multicamadas}

A espessura dos papéis multicamadas produzidos foi medida usando um micrômetro Regmed (modelo ESP / SA-10, Brasil) de acordo com a norma ASTM D645-Método padrão de 97 (ASTM, 2007).

Para análise da gramatura ASTM D646-96 (ASTM, 1996) foram confeccionados corpos de prova circulares de $16 \mathrm{~mm}$ de diâmetro de cada tratamento, onde foram secos em estufa à $50^{\circ} \mathrm{C}$ por $48 \mathrm{~h}$ e pesados. O valor de sua massa foi dividido pelo valor de sua área, conforme Eq. 1:

$$
g=\frac{m}{A}
$$

onde: $\mathrm{g}=$ gramatura $\left(\mathrm{g} / \mathrm{m}^{2}\right) ; \mathrm{m}=$ massa $(\mathrm{g})$ e $\mathrm{A}=$ área do corpo de prova $\left(\mathrm{m}^{2}\right)$

\section{Caracterização morfológica do papel referência e dos papéis multicamadas}

A superfície e a seção transversal dos papéis multicamadas foram avaliadas por microscopia eletrônica de varredura (MEV) usando um microscópio Zeiss Modelo DSM 940 a , pertencente ao Laboratório de Fitopatologia - UFLA. As amostras foram revestidas com ouro por pulverização catódica.

\section{Determinação das propriedades de barreira ao vapor d'água}

As medidas da taxa de transmissão de vapor de água (TPVA) foram realizadas de acordo com o método ASTM E96 /E96M-16 (ASTM, 2016) padrão, conforme utilizado por Guimarães Júnior et al. (2015). Foram confeccionados corpos de prova de 1,6 cm de diâmetro e acoplados a células de permeação, preenchidas com sílica, essas células foram, então, colocados em ambiente com 75\% de UR a $20^{\circ} \mathrm{C}$. Os resultados são a média de três amostras por tratamento, onde as mudanças de massa foram monitoradas durante 8 dias.

A taxa de permeabilidade (TPVA) e a permeabilidade ao vapor de água (PVA) serão calculadas através das Eqs. 3 e 4:

$$
T P V A=\frac{w}{t . A}
$$

onde: TPVA = taxa de permeabilidade ao vapor de água, onde: $\mathrm{w}=$ massa (g) da célula de medida; $\mathrm{t}=$ tempo $(\mathrm{h}) ; \mathrm{A}=$ área exposta do filme $(\mathrm{m})$. A relação w/t será calculada por regressão linear dos pontos experimentais de ganho de massa (g) do filme em função do tempo (horas). 


$$
P V A=\frac{T P V A \cdot e}{p \cdot(U R f-U R d)} \cdot 100
$$

onde: PVA: permeabilidade ao vapor d'água, onde: TPVA = taxa de permeabilidade ao vapor de água; $\mathrm{e}=$ espessura do filme $(\mathrm{mm})$ na célula de medida, $\mathrm{p}=$ pressão de vapor da água $(2,07 \mathrm{kPa})$ e URf - URd é a diferença entre a umidade fora e dentro do recipiente a $25^{\circ} \mathrm{C}$.

\section{RESULTADOS E DISCUSSÃO}

\section{Avaliação da estabilidade das emulsões de ceras}

Nenhuma das formulações empregadas no estudo apresentou separação de fases (coalescência, cremeação ou floculação) após o teste de centrifugação, e depois de um dia da sua preparação (Figura 2) mostrando que ambas se apresentaram estáveis.

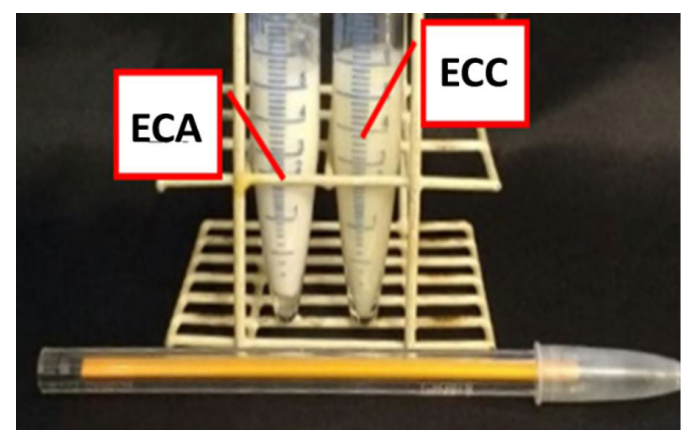

Figura 2: Amostras de emulsão de cera de abelha (ECA) e emulsão de cera de carnaúba (ECC) após os 3 ciclos de centrifugação um dia depois da síntese.

Figure 2: Samples of beeswax emulsion (ECA) and carnauba wax emulsion (ECC) after 3 cycles of centrifugation one day after the synthesis.

Porém, quando o teste foi refeito após 30 dias, observou-se a separação de fases em ECA, onde a proporção da fase aquosa separada, indicada pela seta, foi aumentando com o avanço dos ciclos de centrifugação, conforme Figuras 3a, 3b e 3c. A separação da fase aquosa depois do último ciclo foi de aproximadamente $30 \%$. Desse modo, constatou- se a estabilidade de ECC por um longo período de tempo, o que não aconteceu com ECA.
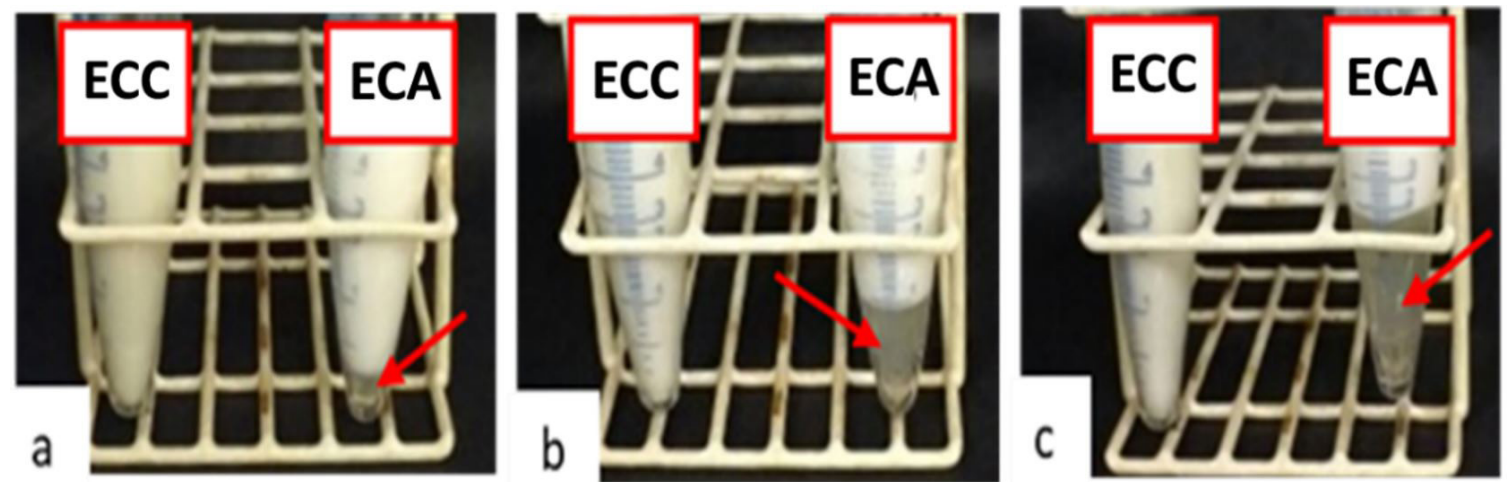

Figura 3: Amostras de emulsão de cera de carnaúba (ECC) e emulsão de cera de abelha (ECA) após os ciclos de 1600 rpm (a), $2400 \mathrm{rpm}$ (b) e 3200 rpm (c) 30 dias após a síntese.

Figure 3: Emulsion samples of carnauba wax (ECC) and beeswax emulsion (ECA) after the cycles of $1600 \mathrm{rpm}$ (a), $2400 \mathrm{rpm}$ (b) and $3200 \mathrm{rpm}$ (c) 30 days after the synthesis.

O método de equilíbrio hidrofílico-lipofílico (EHL) mostrou-se eficiente para a síntese da emulsão de cera de carnaúba, já que ele parte do princípio que a emulsificação adequada se dá quando o agente emulsificante possui EHL igual ao da fase oleosa a ser emulsionada (AULTON, 2005). 


\section{Espessura e gramatura}

Como esperado, a espessura e a gramatura de todos os tratamentos aumentaram em relação ao papel $\mathrm{kraft}$ de $60 \mathrm{~g} / \mathrm{m}^{2}$ (Tabela 2), pois foram depositadas camadas de revestimento sobre ele. Porém, quando comparados ao papel kraft de $80 \mathrm{~g} / \mathrm{m}^{2}$ apenas MMT/EA, NF e NF/EA apresentaram valores de espessura igual ou superior a Ref 80, sendo que o revestimento com nanofibrilas (NF) atingiu o maior valor $(0,113 \mathrm{~mm})$. Segundo Brodin et al. (2014), esse é um resultado positivo, pois para alterar propriedades de barreira do papel são necessárias camadas mais contínuas e densas de nanofibrilas celulósicas sobre o papel.

Tabela 2: Tratamentos, número de camadas, valores médios e desvios padrão da espessura e gramatura dos papéis multicamadas

Table 2: Treatments, number of layers, mean values and standard deviations of thickness and weight of multilayer papers

\begin{tabular}{cccc}
\hline TRATAMENTO & $\mathbf{N}^{\circ}$ DE CAMADAS & ESPESURA $(\mathrm{mm})$ & GRAMATURA $\left(\mathrm{g} / \mathbf{m}^{2}\right)$ \\
\hline Ref 60 & 1 & $0,082 \pm 0,003$ & $63,0 \pm 0,9$ \\
Ref 80 & 1 & $0,110 \pm 0,004$ & $84,1 \pm 0,9$ \\
EA & 2 & $0,106 \pm 0,001$ & $87,8 \pm 0,9$ \\
EC & 2 & $0,104 \pm 0,003$ & $87,7 \pm 0,9$ \\
MMT & 2 & $0,104 \pm 0,002$ & $80,7 \pm 1,4$ \\
MMT/EA & 3 & $0,111 \pm 0,003$ & $91,3 \pm 0,4$ \\
MMT/EC & 3 & $0,099 \pm 0,005$ & $85,2 \pm 1,3$ \\
NF & 2 & $0,113 \pm 0,001$ & $83,3 \pm 0,6$ \\
NF/EA & 3 & $0,110 \pm 0,004$ & $86,0 \pm 1,6$ \\
NF/EC & 3 & $0,102 \pm 0,002$ & $86,2 \pm 0,9$ \\
\hline
\end{tabular}

Ref 60: papel kraft $60 \mathrm{~g} / \mathrm{m}^{2}$; Ref 80: papel kraft $80 \mathrm{~g} / \mathrm{m}^{2}$; EA: papel kraft $0 \mathrm{~g} / \mathrm{m}^{2}$ com emulsão de cera de abelha; EC: papel kraft $60 \mathrm{~g} / \mathrm{m}^{2} \mathrm{com}$ emulsão de cera de carnaúba; MMT: papel kraft $60 \mathrm{~g} / \mathrm{m}^{2} \mathrm{com}$ montimorilonita; MMT/EA: papel kraft $60 \mathrm{~g} / \mathrm{m}^{2} \mathrm{com}$ montimorilonita e emulsão de cera de abelha; MMT/EC: papel kraft $60 \mathrm{~g} / \mathrm{m}^{2} \mathrm{com}$ montimorilonita e emulsão de cera de carnaúba; NF: papel $\mathrm{kraft} 60 \mathrm{~g} / \mathrm{m}^{2} \mathrm{com}$ nanofibrilas celulósicas; NF/EA: papel kraft $60 \mathrm{~g} / \mathrm{m}^{2} \mathrm{com}$ nanofibrilas celulósicas e emulsão de cera de abelha; NF/EC: papel $\mathrm{kraft} 60 \mathrm{~g} / \mathrm{m}^{2} \mathrm{com}$ nanofibrilas celulósicas e emulsão de cera de carnaúba.

Todos os tratamentos de papéis revestidos apresentaram gramaturas próximas a Ref 80, exceto MMT/EA, que apresentou valor muito acima $\left(\sim 92 \mathrm{~g} / \mathrm{m}^{2}\right)$. Verificou-se que as emulsões de ceras de abelha e carnaúba conferiram maior gramatura aos papéis, cerca de $36 \%$ a $44 \%$ acima da encontrada para Ref 60 e cerca de $2 \%$ a $13 \%$ acima da encontrada para Ref 80 . Gramatura e espessura são propriedades importantes dos papéis multicamadas, visto que, uma camada fina de material nanométrico pode ser suficiente para mudar grandemente suas propriedades de superfície.

\section{Caracterização da microestrutura dos papéis multicamadas Superfície dos papéis multicamadas}

As micrografias apontaram presença de muitos espaços vazios na superfície do papel kraft de $60 \mathrm{~g} / \mathrm{m}^{2}$, porém quando depositadas as camadas sobre ele as imagens de MEV indicaram redução desses espaços vazios em todos os tratamentos (Figuras 4 a 12).
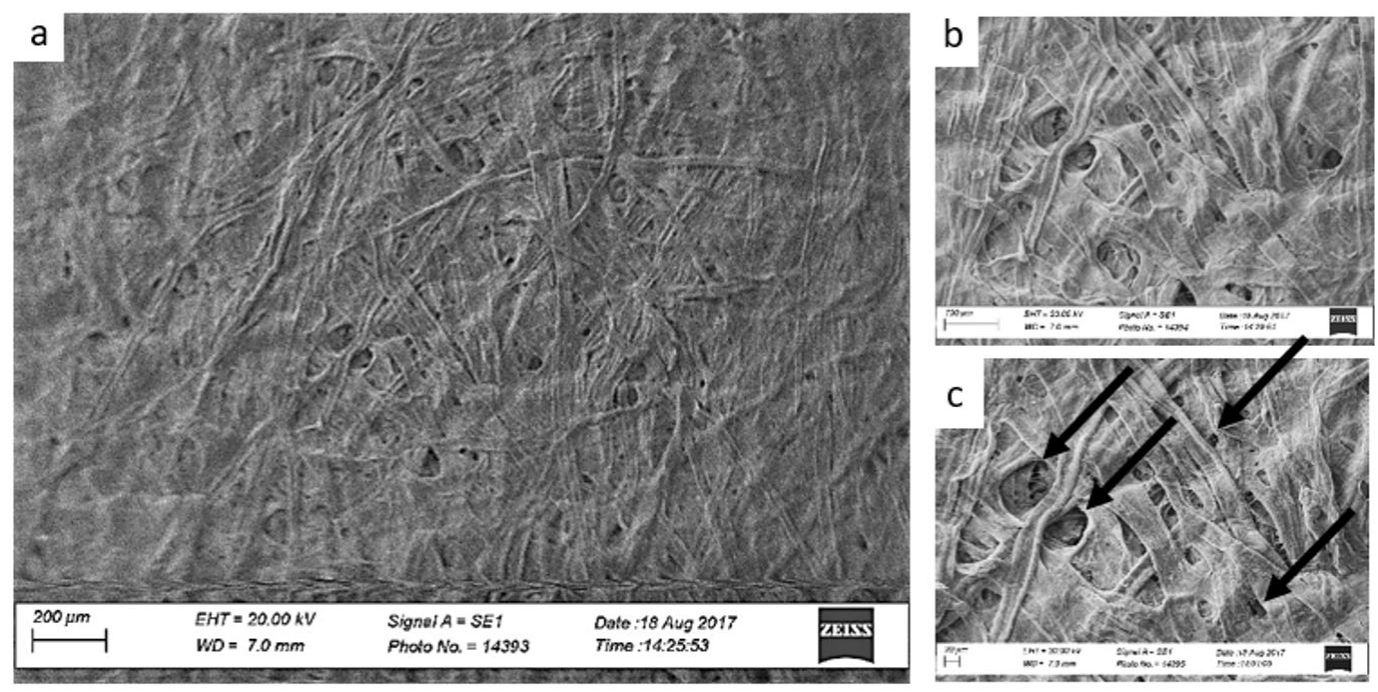

Figura 4: Micrografias de MEV da superfície do papel kraft referência (sem revestimento) de $60 \mathrm{~g} / \mathrm{m}^{2}$ (Ref 60)

Figure 4: SEM micrographs of the reference kraft paper surface (uncoated) of $60 \mathrm{~g} / \mathrm{m}^{2}$ (Ref 60) 
Matos et al. - Incorporação de Nanomateriais e emulsão de ceras no

desenvolvimento de papéis multicamadas
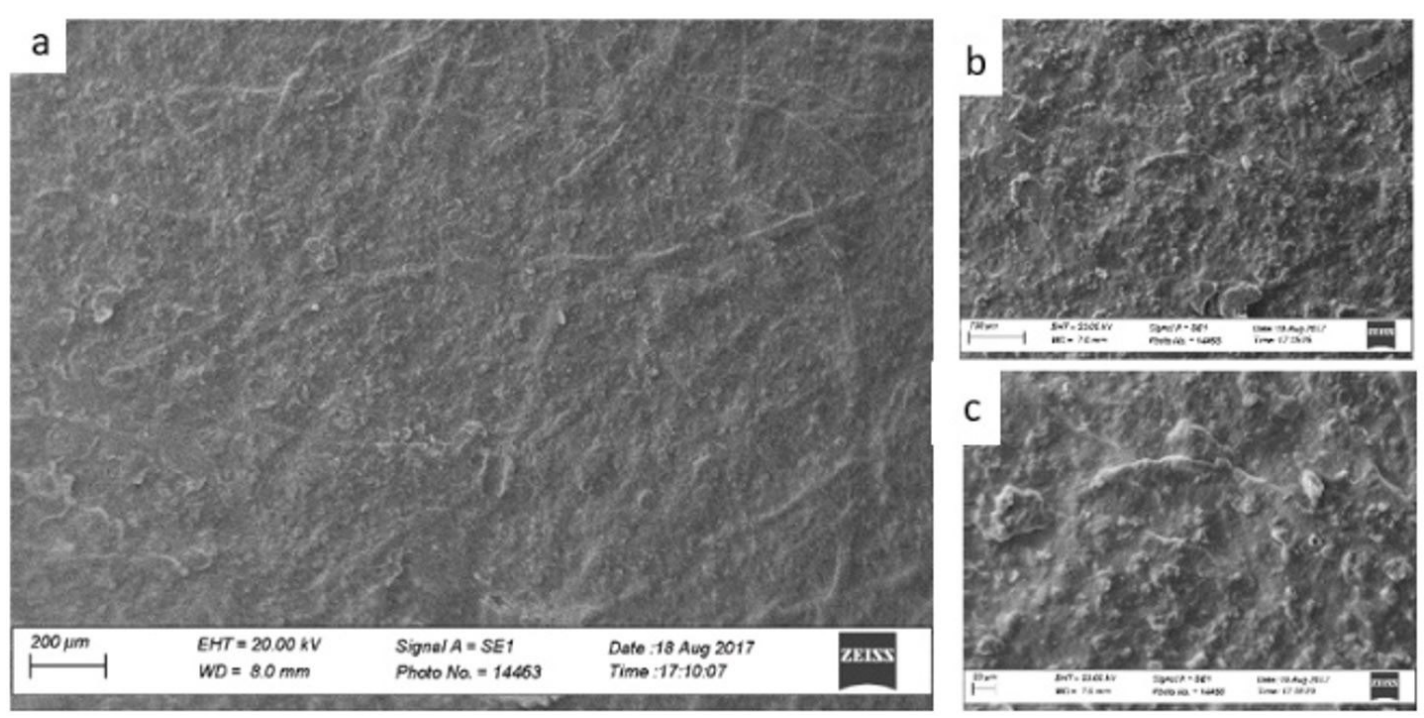

Figura 5: Micrografias de MEV da superfície do papel kraft com uma camada de emulsão de cera de abelha (EA)

Figure 5: SEM micrographs of the surface of the kraft paper with a layer of emulsion of beeswax (EA)
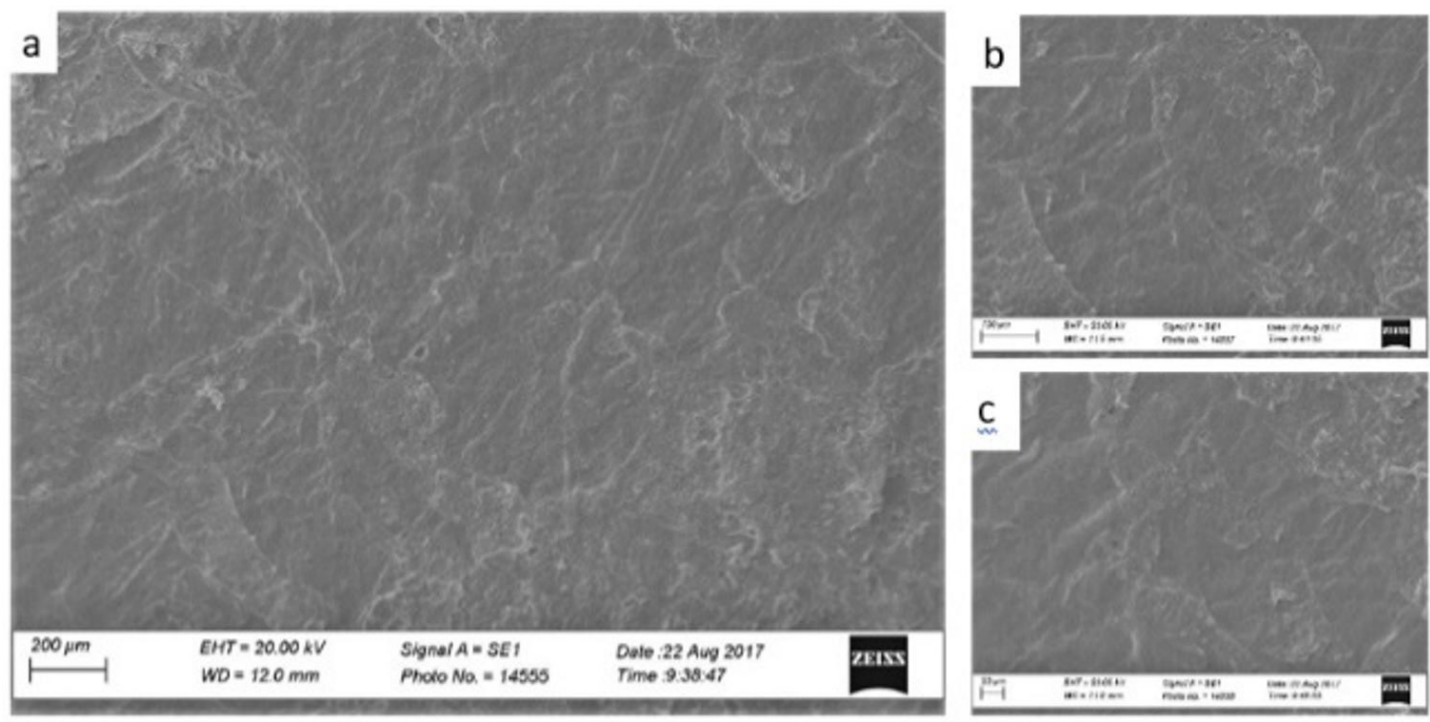

Figura 6: Micrografias de MEV da superfície do papel kraft com uma camada de emulsão de cera de carnaúba (EC) Figure 6: SEM micrographs of the surface of kraft paper with a layer of emulsion of carnauba wax (EC)
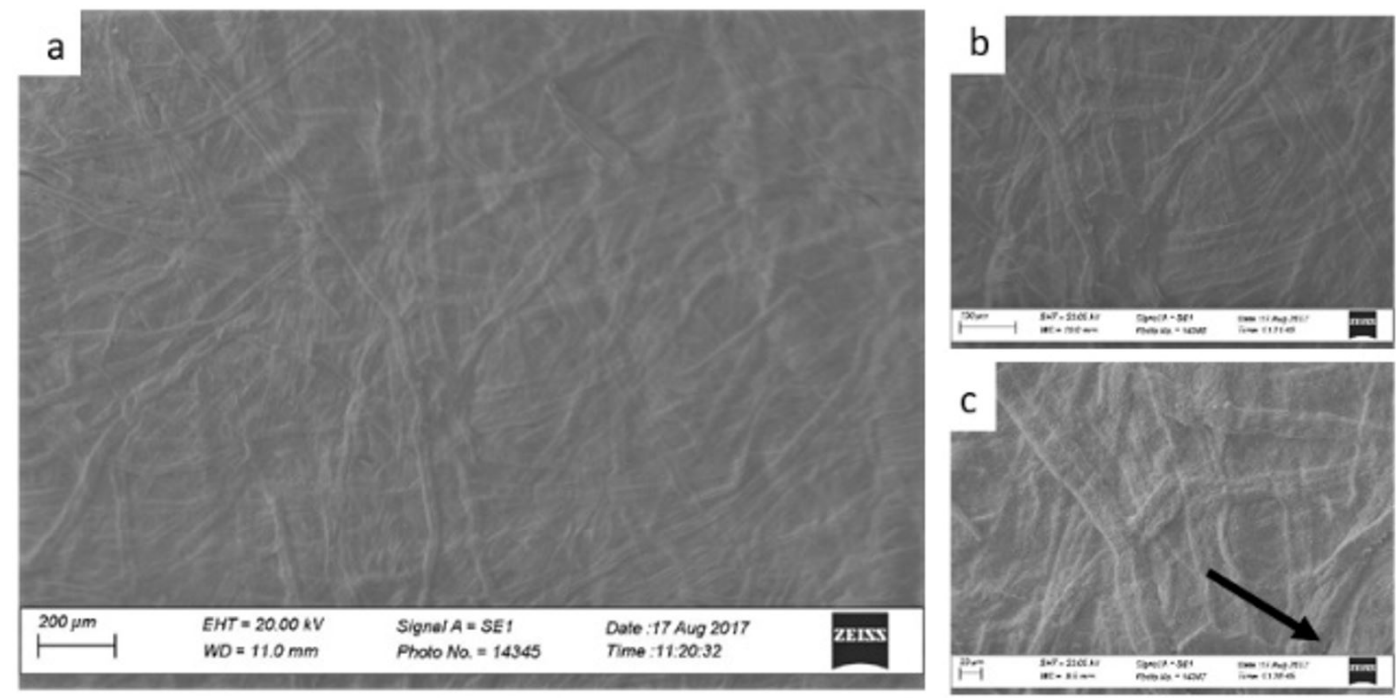

Figura 7: Micrografias de MEV da superfície do papel kraft com uma camada de montimorilonita(MMT) Figure 7: SEM micrographs of the surface of the kraft paper with a layer of montimorilonite (MMT) 

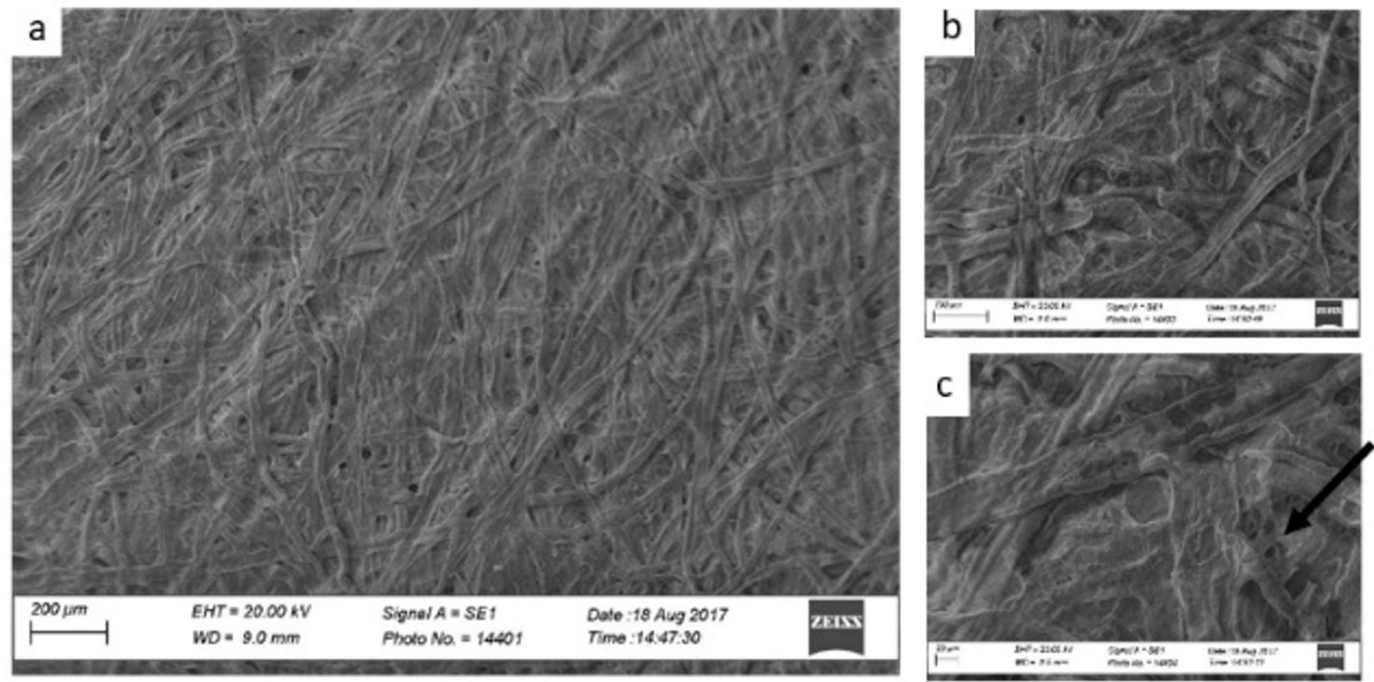

Figura 8: Micrografias de MEV da superfície do papel kraft com uma camada de montimorilonita e uma de emulsão de cera de abelha (MMT/EA)

Figure 8: SEM micrographs of the surface of the kraft paper with a montimorilonite layer and a beeswax emulsion (MMT / EA)
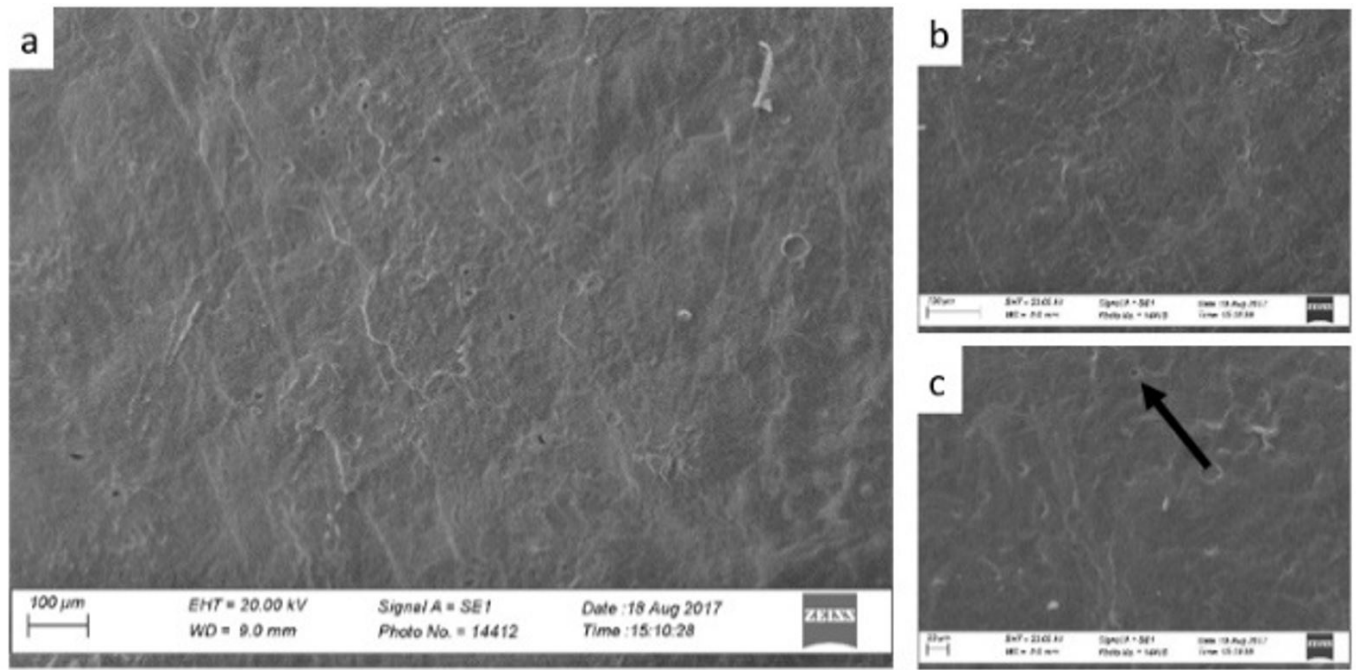

Figura 9: Micrografias de MEV da superfície do papel kraft com uma camada de montimorilonita e uma de emulsão de cera de carnaúba (MMT/EC)

Figure 9: SEM micrographs of the surface of the kraft paper with a layer of montimorilonite and one of carnauba wax emulsion (MMT / EC)
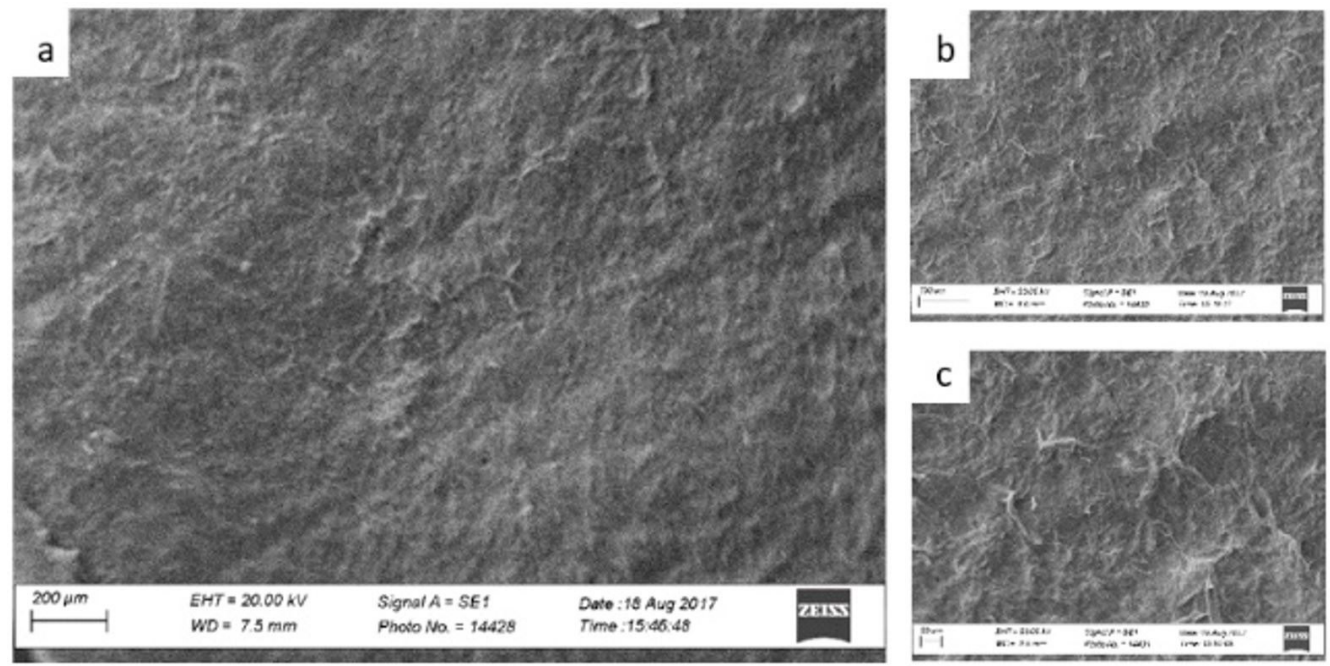

Figura 10: Micrografias de MEV da superfície do papel kraft com uma camada de nanofibrilas celulósicas (NF) Figure 10: SEM micrographs of the kraft paper surface with a layer of cellulosic 

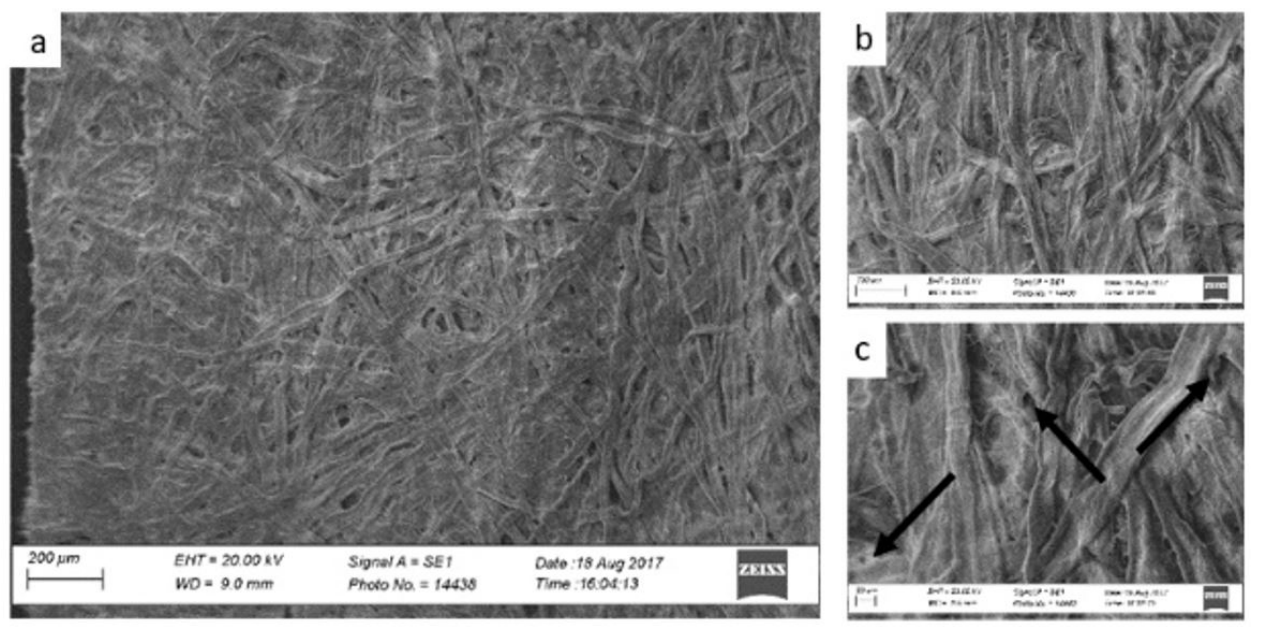

Figura 11: Micrografias de MEV da superfície do papel kraft com uma camada de nanofibrilas celulósicas e uma de emulsão de cera de abelha (NF/EA)

Figure 11: SEM micrographs of the kraft paper surface with a layer of cellulosic nanofibrils and a beeswax emulsion (NF / EA)
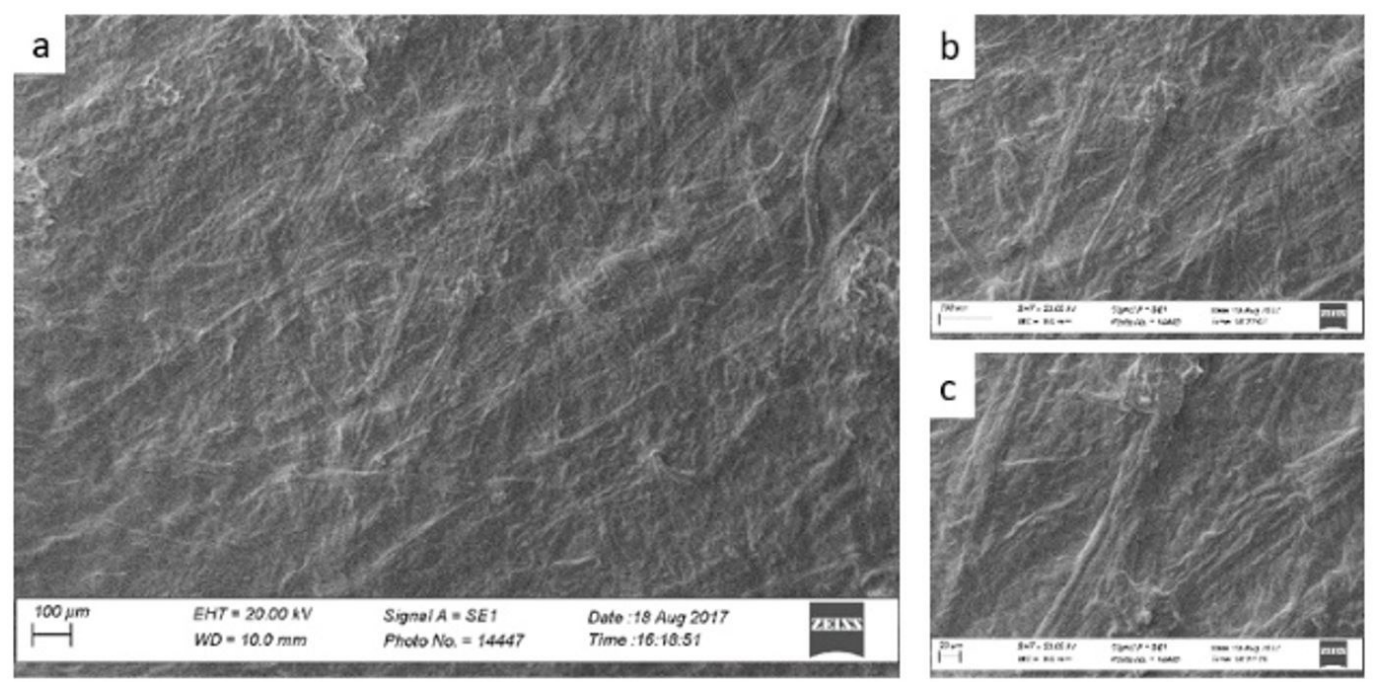

Figura 12: Micrografias de MEV da superfície do papel kraft com uma camada nanofibrilas celulósicas e uma de emulsão de cera de carnaúba (NF/EC)

Figure 12: SEM micrographs of the surface of the kraft paper with a layer of cellulosic nanofibrils and one of carnauba wax emulsion (NF / EC)

Nos tratamentos com emulsão de cera de abelha (Figuras 5, 8 e 11) ainda é possível ver a incidência de espaços vazios em MMT/EA e NF/EA, indicados pelas setas, sendo maior a ocorrência de espaços vazios em NF/EA. Comparando os papéis multicamadas com cera de abelha verifica-se que a incidência de espaços vazios na superfície diminui. Nesses tratamentos a hidrofobicidade da cera aplicada e a redução dos espaços vazios fez com que a interação da água com a superfície do papel fosse reduzida.

Nos tratamentos restantes, EC; MMT; MMT/EC; NF e NF/EC; (Figuras 6, 7, 9, 10 e 12), observa-se a presença de espaços vazios apenas nos dois últimos, com poros em tamanhos menores e em menor quantidade do que em Ref 60. Ainda pelas imagens de MEV da superfície dos papéis é possível observar a homogeneidade nas camadas depositadas sobre o papel de $60 \mathrm{~g} / \mathrm{m}^{2}$, indicando eficiência no método de deposição de camadas por espatulagem usado para a formação das camadas sobre o papel.

\section{Seção transversal dos papéis multicamadas}

As micrografias da seção transversal dos papéis multicamadas também mostram que os espaços vazios presentes em Ref 60 diminuíram com a incorporação das camadas de nanofibrilas celulósicas, montimorilonita e emulsões de ceras de carnaúba e de abelha (Figura 13), como visto anteriormente nas micrografias de MEV da superfície. 



Figura 13: Micrografias de MEV da seção transversal de Ref 60 (a), EA (b), EC (c), MMT (d), MMT/EA (e), MMT/EC (f), NF (g), NF/EA (h) e NF/EC (i)

Figure 13: SEM micrographs of the cross section of Ref 60 (a), EA (b), EC (c), MMT (d), MMT / EA (e), MMT / EC NF / EA (h) and NF / EC (i) 
Em algumas das imagens ainda é possível observar as camadas sobre a superfície de Ref 60, indicadas pelas setas nas Figuras 13c, 13g, 13h e 13i. Nas micrografias de NF e NF/EC, Figuras 13g e 13i, a camada de nanofibrilas celulósicas e de nanofibrilas celulósicas sobreposta por emulsão de carnaúba aparecem bem definidas e compactas sobre o substrato de papel. Ainda é possível observar, pelas micrografias da seção transversal dos papéis multicamadas, que as suspensões de revestimento penetraram pelo substrato, preenchendo seus espaços vazios, conforme indicado pela seta na Figura $13 \mathrm{e}$.

\section{Propriedades de barreira ao vapor d'água dos papéis multicamadas}

Na Tabela 3 estão os resultados encontrados para a taxa de permeabilidade ao vapor d'água (TPVA) e permeabilidade ao vapor d'água (PVA) dos papéis referência e dos papéis multicamadas. A adição das camadas de nanofibrilas celulósicas (NF), montimorilonita (MMT) e emulsões de ceras de carnaúba (ECC) e de abelha (ECA) causou redução de TPVA e PVA em relação a referência (sem revestimento) com a mesma gramatura (Ref 80). Nos tratamentos com camadas de ECC e ECA observou-se redução de $87 \%$ e 69\%, respectivamente em PVA em relação a Ref 60, e quando comparados com Ref 80 a redução de PVA foi ainda maior, de 96\% para EC e 90\% para EA.

Tabela 3: Valores médios e de desvio-padrão para TPVA e PVA para os tratamentos estudados.

Table 3: Mean and standard deviation values for TPVA and PVA for the treatments studied.

\begin{tabular}{ccc}
\hline TRATAMENTOS & TPVA $\left(\mathbf{g} / \mathbf{m}^{2} \cdot \mathbf{d}\right)$ & PVA $\left(\mathbf{g} \cdot \mathbf{m m} / \mathbf{d i a} \cdot \mathbf{m}^{2} . \mathbf{K p a}\right)$ \\
\hline Ref 60 & $222,8 \pm 15,0$ & $11,1 \pm 1,4$ \\
Ref 80 & $502,7 \pm 11,8$ & $37,4 \pm 0,9$ \\
EA & $58,9 \pm 5,0$ & $3,4 \pm 1,0$ \\
EC & $20,4 \pm 3,1$ & $1,4 \pm 0,5$ \\
MMT & $255,5 \pm 3,8$ & $17,0 \pm 0,3$ \\
MMT/EA & $59,8 \pm 4,1$ & $4,3 \pm 1,1$ \\
MMT/EC & $46,3 \pm 2,2$ & $2,6 \pm 1,3$ \\
NF & $315,0 \pm 23,7$ & $24,8 \pm 2,8$ \\
NF/EA & $79,8 \pm 6,8$ & $5,1 \pm 1,1$ \\
NF/EC & $107,8 \pm 12,7$ & $6,8 \pm 0,8$
\end{tabular}

Ref 60: papel kraft $60 \mathrm{~g} / \mathrm{m}^{2}$; Ref 80: papel kraft $80 \mathrm{~g} / \mathrm{m}^{2}$; EA: papel $\mathrm{kraft} 60 \mathrm{~g} / \mathrm{m}^{2}$ com emulsão de cera de abelha; EC: papel kraft $60 \mathrm{~g} / \mathrm{m}^{2} \mathrm{com}$ emulsão de cera de carnaúba; MMT: papel $\mathrm{kraft} 60 \mathrm{~g} / \mathrm{m}^{2} \mathrm{com}$ montimorilonita; MMT/EA: papel $\mathrm{kraft} 60 \mathrm{~g} / \mathrm{m}^{2} \mathrm{com}$ montimorilonita e emulsão de cera de abelha; MMT/EC: papel $\mathrm{kraft} 60 \mathrm{~g} / \mathrm{m}^{2} \mathrm{com}$ montimorilonita e emulsão de cera de carnaúba; NF: papel $\mathrm{kraft} 60 \mathrm{~g} / \mathrm{m}^{2} \mathrm{com}$ nanofibrilas celulósicas; NF/EA: papel $\mathrm{kraft} 60 \mathrm{~g} / \mathrm{m}^{2} \mathrm{com}$ nanofibrilas celulósicas e emulsão de cera de abelha; NF/EC: papel $\mathrm{kraft} 60 \mathrm{~g} / \mathrm{m}^{2} \mathrm{com}$ nanofibrilas celulósicas e emulsão de cera de carnaúba

Os menores valores de TPVA e PVA foram observados para EC devido à ausência de espaços vazios e homogeneidade na superfície da embalagem multicamada, que diminuiu a permeação de gases, o que pode ser observado nas imagens da microestrutura (Figura 6).

Nos tratamentos com camadas de nanomateriais adicionados das camadas de emulsão das ceras foi observado maior redução de PVA nos tratamentos com montimorilonita (76\% de redução para MMT/EA e 60\% para MMT/EC), do que nos tratamentos com nanofibrilas celulósicas (38\% de redução para NF/EA e 53\% para NF/EC). Nos tratamentos com montimorilonita, o caminho tortuoso criado em torno das placas de nanoargila forçou o vapor de água a percorrer um caminho mais longo para se difundir através dos papéis multicamadas, o que foi potencializado pela associação com as camadas de emulsão das ceras. Resultados similares foram observados por Sothornvit (2009) com a redução de PVA em papel revestido com HPMC e cera de abelha, enquanto que Zhang et al. (2014) observaram redução de 69\% na TPVA de papéis revestidos por quitosana e cera de abelha. Despond et al. (2005) observaram que a absorção de água em papel revestido com quitosana diminuiu ainda mais com o uso de uma camada de cera de carnaúba sobre a camada de quitosana. Mirmehdi et al. (2018) também reportaram diminuição da permeabilidade a gases de papel de escrita revestidos com nanoargila e nanofibrilas.

Os valores de TPVA e PVA dos tratamentos cujo o papel de $60 \mathrm{~g} / \mathrm{m}^{2}$ foi revestido apenas com camadas de nanofibrilas celulósicas (NF) e de montimorilonita (MMT) podem estar associados à hidrofilicidade dessas nanoestruturas e a menor quantidade de material depositada sobre o papel, visto que estes foram os tratamentos que atingiram a menor gramatura ( 83 e $81 \mathrm{~g} / \mathrm{m}^{2}$, respectivamente). Futuros trabalhos podem explorar o uso de camadas de NF e MMT mais espessas para aumentar o caminho tortuoso e aumentar a barreira a passagem de gases. Nas micrografias de MMT e NF (Figuras 7 e 10) a baixa espessura das camadas de MMT e NF permite ainda identificar a silhueta das fibras que compõe o papel substrato. 


\section{CONCLUSÕES}

Os papéis revestidos com nanofibrilas celulósicas, nanoargila (montmorilonita) e emulsões de ceras foram mais eficientes quanto a barreira ao vapor de água quando comparados aos não revestidos. A incorporação de emulsão de ceras de carnaúba e de abelha no papel reduz a permeabilidade ao vapor de água (PVA) do papel de $60 \mathrm{~g} / \mathrm{m}^{2}$ em $87 \%$ e 96\%, respectivamente. Em relação ao papel de $80 \mathrm{~g} / \mathrm{m}^{2}$, ocorreu a redução de aproximadamente $68 \%$ e $90 \%$ da PVA para os papéis revestidos com emulsão de cera de carnaúba e de abelha, respectivamente. Os valores de permeabilidade (TPVA e PVA) de papéis com gramatura de $80 \mathrm{~g} / \mathrm{m} 2$ foram menores em papéis revestidos com nanofibrilas celulósicas quando comparados aos valores encontrados em papéis comerciais com fibras convencionais. As imagens de MEV da superfície dos papéis multicamadas e na seção transversal mostraram que a adição de nanofibrilas celulósicas, montimorilonita e emulsão de ceras de carnaúba e abelha reduzem a ocorrência de espaços vazios na microestrutura do papel.

Este trabalho contribui com a evolução no uso de nanofibrilas celulósicas para obtenção de papéis multicamadas de alto desempenho para embalagens. Mostra também o alto potencial das ceras de carnaúba e abelha como revestimento para melhoria das propriedades de barreira ao vapor de água de papéis multicamadas. Adicionalmente, colabora com informações sobre a combinação de camadas de nanomateriais (nanofibrilas de celulose e montimorilonita) e camadas de emulsão de ceras para melhorar o desempenho dos papéis para embalagens mais renováveis.

\section{REFERÊNCIAS BIBLIOGRAFICAS}

ALMEIDA, M. L. Estabilidade de emulsões de água-em-óleo na presença de campo elétrico externo. 103 p. 2014. Dissertação (Mestrado em Engenharia Química) - Universidade Federal do Rio de Janeiro, Rio de Janeiro, 2014.

ALVES, M. O.; COÊLHO, J. D. Extrativismo da carnaúba: relações de produção, tecnologia e mercados. Fortaleza: Banco do Nordeste do Brasil, 2008.

ASTM - AMERICAN SOCIETY FOR TESTING AND MATERIALS. E96 / E96M -16: Standard Test Method for Water Vapor Transmission of Material. Philadelphia, 2016. 14 p.

ASTM - AMERICAN SOCIETY FOR TESTING AND MATERIALS. ASTM D645/D645M - Standard Test Method for Thickness of Paper and Paperboard. Philadelphia, 2007. 3 p.

ASTM - AMERICAN SOCIETY FOR TESTING AND MATERIALS. ASTM D646 - 96 - Standard Test Method for Grammage of Paper na Paperboard (Mass Per United Area) Philadelphia, 1996. 5 p.

AMINI, ELAHE;AZADFALLAH, M.; LAYEGHI, M.; REZA, T. H. Silver-nanoparticle-impregnated cellulose nanofiber coating for packaging paper. Cellulose, v. 23, n. 1, p. 557-570, 2016.

ARANTES, A. C. C.; ALMEIDA, C.; DAUZACKER, L.; BIANCHI, M. L.; WOOD, D.; WILLIAMS T; ORTS, W. J.; TONOLI, G.H.D. Renewable hybrid nanocatalyst from magnetite and cellulose for treatment of textile effluents. Carbohydrate Polymers, v. 163, p. 101 - 107, 2017.

BANDERA, Davide et al. Polylactide/Montmorillonite hybrid latex as a barrier coating for paper applications. Polymers, v. 8, n. 3, p.75, 2016.

BUFALINO, L.; SENA NETO, A. R.; TONOLI, G. H. D.; FONSECA, A. S.; COSTA, T. G.; MARCONCINI, J. M.; COLODETTE, J. L.; LABORY, C. R. G.; MENDES, L. M. How the chemical nature of Brazilian hardwoods affects nanofibrillation of cellulose fibers and film optical quality. Cellulose, v. 22, p. 3657-3672, 2015.

D’ALVA, O. A. O extrativismo da carnaúba no Ceará. Fortaleza: Banco do Nordeste do Brasil, p. 172, 2007.

DESPOND, S.; ESPUCHE, N.; CARTIER, N.; DOMARD, A. Barrier properties of paper-chitosan and paperchitosan-carnauba wax films. Journal Applied Polymer Science, v. 98, p. 704-10, 2005.

FLOODY, M. C.; THENG, B. K. G.; MORA, M. L. Natural nanoclays: applications and future trends - a Chilean perspective. Clay Minerals, v. 44, n. 2, p.161-176, 2009. 
Matos et al. - Incorporação de Nanomateriais e emulsão de ceras no

desenvolvimento de papéis multicamadas

FONSECA, C. S.; SILVA, T. F.; SILVA, M. F.; OLIVEIRA, I. R. C.; MENDES, R. F.; HEIN, P. R. G.; MENDES, L. M.; TNOLI, G. H. D. Micro/nanofibrilas celulósicas de eucalyptus em fibrocimentos estruturados. Cerne, Lavras, v. 22, p. 59-68, 2016.

GUIMARÃES JÚNIOR, M.; TEIXEIRA, F.G.; TONOLI, G.H.D.; NOVACK, K. M Effect of the nano-fibrillation of bamboo pulp on the thermal, structural, mechanical and physical properties of nanocomposites based on starch/poly(vinyl alcohol) blend. Cellulose v. 25, n. 3, p. 1-27, 2018.

GUIMARÃES JÚNIOR, M.; BOTARO, V. R.; NOVACK, K. M.; FLAUZINO NETO, W. P.; MENDES, L. M.; TONOLI, G. H. D. Preparation of celulose nanofibrils from bamboo pulp by mechanical defibrillation for their applications in biodegradable composites. Journal of Nanoscience and Nanotechnology, v. 15, p. 6751-6768, 2015.

HERRERA, M. A.; MATHEW, A. P.; OKSMAN, K. Gas permeability and selectivity of cellulose nanocrystals films (layers) deposited by spin coating. Carbohydrate Polymers, v. 112, p.494-501, 2014.

HULT, E.; IOTTI, M.; LENES, M. Efficient approach to high barrier packaging using microfibrillar cellulose and shellac. Cellulose, v. 17, p.575-586. 2010

JO, W.; BUM, N.; HYUN, J. Quality and microbial safety of Fuji apples coated with carnauba-shellac wax containing lemongrass oil. Food Science and Technology, v. 55, n. 2, p. 490-497, 2014.

KK, G.; KO, S. Overview on in polymer-nano clay composite paper coating for packaging application. Journal of Material Science \& Engineering, Libertyville, v. 4, p. 151, 2015.

LI, X.; TABIL, L. G.; PANIGRAHI, S. Chemical Treatments of Natural Fiber for Use in Natural Fiber-Reinforced Composites: A Review. Journal of Polymer and the Environment, v. 15, n.1, p. 25- 33, 2007.

MIRMEHDI, S.; HEIN, P.R.G.; SARANTOPOULOS, C. I. G. L.; DIAS, M. V.; TONOLI, G. H. D. Cellulose nanofibrils/nanoclay hybrid composite as a paper coating: Effects of spray time, nanoclay content and corona discharge on barrier and mechanical properties of the coated papers. Food Packaging and Shelf Life, v. 15, p. 87-94, 2018.

MOUSAVI, S. M. Mazhari et al. Cellulose nanofiber/carboxymethyl cellulose blends as an efficient coating to improve the structure and barrier properties of paperboard. Cellulose, v. 24, n. 7, p. 3001-3014, 2017.

OJIJO, V.; RAY, S. S. Processing strategies in bionancomposites. Progress in Polymer Science, v. 38, p. 1543$1589,2013$.

OLIVEIRA, V. R. L. E. Avaliação da incorporação de cera de abelha na hidrofobização de filmes biopoliméricos e seu efeito como cobertura na conservação pós-colheita da goiaba - Dissertação (Mestrado em Engenharia de Materiais) - Universidade Federal Rural do Semiárido, Mossoró, 2017.

OURIQUE, P. A.; CRUZ, R. C. D.; ZORZI, J. E. Influência da cera de carnaúba no comportamento reológico de misturas usadas na moldagem por injeção em baixa pressão. Cerâmica, v. 61, n. 357, p.71-76, 2015.

PRADO, N. R. T.; RAABE, J.; MIRMEHDI, S., HUGEN, L. N., LIMA, L. C., RAMOS, A. L. S., GUIMARÃES JUNIOR, M., TONOLI, G. H. D. Strength improvement of hydroxypropyl methylcellulose/ starch films using cellulose nanocrystals. Cerne, v. 23, n. 4, p. 423-434, 2018.

SCATOLINO, M. O. V.; SILVA, D. W.; BUFALINO, L.; DENZIN TONOLI, G. H.; MENDES, L. M. Influence of cellulose viscosity and residual lignin on water absorption of nanofibril films. Procedia Engineering, v. 200, p. $155-161,2017$.

SILVA, D. L. Influência da adição de nanocristais e nanofibras de celulose extraídas de bagaço de cana de açúcar nas propriedades de embalagens biodegradáveis. 2016. 168 p. Tese (Doutorado em Engenharia de Alimentos) - Universidade Federal de Viçosa, Viçosa, 2016.

SOTHORNVIT, R. Effect of hydroxypropyl methylcellulose and lipid on mechanical properties and water vapor permeability of coated paper. Food Research International, v. 42, n. 2, p. 307, 2009.

TARRÉS, Q.; SAGUER, E. M.; PÈLACH, M.; ALCALÀ, M.; DELGADO-AGUILAR, M. The feasibility of incorporating cellulose micro/nanofibers in papermaking processes: the relevance of enzymatic hydrolysis. Cellulose, v. 23, n. 2, p. 1433-1445, 2016. 
TONOLI, G. H. D.; HOLTMAN, K. M.; GLENN, GREGORY; FONSECA, A. S.; WOOD, D.; WILLIAMS T; SA, V. A.; TORRES, L.; KLAMCZYNSKI, A.; ORTS, W. J. Properties of cellulose micro/nanofibers obtained from eucalyptus pulp fiber treated with anaerobic digestate and high shear mixing. Cellulose, v. 23, p. 1 - 18, 2016.

UGALDE, M. L. Biofilmes ativos com incorporação de óleos essenciais. 2014. 168 p. Tese (Doutorado em Engenharia de Alimentos) - Universidade Regional Integrada do Alto Uruguai, Erechim, 2014.

WANG, S.; JING, Y. Effects of formation and penetration properties of biodegradable montmorillonite/chitosan nanocomposite film on the barrier of package paper. Applied Clay Science, v. 138, p.74-80, 2017.

ZHANG, W.; XIAO, H.; QIAN, L. Enhanced water vapour barrier and grease resistance of paper bilayer-coated with chitosan and beeswax. Carbohydrate Polymers, v. 101, p.401-406, 2014.

Recebido em: 23/01/2018

Aceito em: 03/09/2018 